\title{
Surgical management of abdominal compartment syndrome; indications and techniques
}

\author{
Ari Leppäniemi
}

Address: Department of Surgery, Helsinki University Hospital, Haartmaninkatu 4, PO Box 340, 00029 HUS, Helsinki, Finland

Email: Ari Leppäniemi - ari.leppaniemi@hus.fi

Published: 14 April 2009

Scandinavian Journal of Trauma, Resuscitation and Emergency Medicine 2009, 17:17 doi:10.1 186/1757-724I-

17-17

This article is available from: http://www.sjtrem.com/content//7///17

(C) 2009 Leppäniemi; licensee BioMed Central Ltd.

This is an Open Access article distributed under the terms of the Creative Commons Attribution License (http://creativecommons.org/licenses/by/2.0), which permits unrestricted use, distribution, and reproduction in any medium, provided the original work is properly cited.
Received: 19 February 2009

Accepted: 14 April 2009

\begin{abstract}
The indications for surgical decompression of abdominal compartment syndrome (ACS) are not clearly defined, but undoubtedly some patients benefit from it. In patients without recent abdominal incisions, it can be achieved with full-thickness laparostomy (either midline, or transverse subcostal) or through a subcutaneous linea alba fasciotomy. In spite of the improvement in physiological variables and significant decrease in IAP, however, the effects of surgical decompression on organ function and outcome are less clear. Because of the significant morbidity associated with surgical decompression and the management of the ensuing open abdomen, more research is needed to better define the appropriate indications and techniques for surgical intervention.
\end{abstract}

\section{Introduction}

Sustained increase in intra-abdominal pressure (IAP) defined as intra-abdominal hypertension (IAH) can with ensuing onset of organ dysfunction lead to abdominal compartment syndrome (ACS) $[1,2]$. If not timely treated, it can lead to serious organ failures and even death. Whether the cause is in the abdomen (primary ACS) or elsewhere (secondary ACS), prevention is of utmost importance. Fluid resuscitation is a very important cause of IAH when high volumes are necessary, such as in patients with severe acute pancreatitis (SAP). In these patients, the excessive use of crystalloids should be avoided.

The first line of treatment is always nonoperative [3]. Nasogastric decompression is useful in patients with gastric dilatation or ileus. Short term use of neuromuscular blocking agents has been used in appropriate circumstances. Removal of excessive fluids with diuretics can be tried, but extracorporeal techniques, such as continuous hemodiafiltration, are more effective in rapidly removing excess fluid. Finally, percutaneous drainage of intraperitoneal fluid collections is a simple and more effective way to reduce intra-abdominal volume.

If nonoperative measures fail to relieve ACS, surgical decompression should be considered. Due to the severe morbidity associated with all forms of surgical decompression, the indications, timing and technique used should be carefully evaluated.

\section{Indications for decompression}

There is no uniform consensus on the indications for surgical decompression in ACS. In addition to IAP values, the cause, time-frame and possible need for further laparotomy should be considered. As a general rule, when nonsurgical interventions fail to turn around the progressive deterioration of organ dysfunctions in the presence of fulminate ACS, surgical decompression is justified. 


\section{Timing of decompression}

Clinical experience seems to indicate that early decompression is more effective and associated with lower mortality than delayed decompression performed after several days after the onset of ACS, but no comparative studies exist. Obviously, it depends on the time when ACS develops. Usually ACS is an early phenomenon and prompt decompression without significant delay is appropriate. However, if ACS is caused by a later event, such as infection of the peripancreatic necrosis in SAP, for example, decompression at that stage is warranted and often combined with necrosectomy. There are no studies on prophylactic surgical decompression, but preventing ACS by leaving the abdomen open in high risk patients, such as patients undergoing damage control surgery for trauma or patients operated for ruptured abdominal aortic aneurysms, is sensible.

\section{Techniques}

When selecting the optimal technique for surgical decompression, previous already existing recent abdominal incisions (wounds) are preferable. If a patient has undergone a midline laparotomy, utilizing the same incision for decompression is obviously the best choice. In patients with secondary ACS and particularly those without previous abdominal incisions, several options exist. The most commonly used method for surgical decompression is the midline laparostomy [4]. All layers (skin, fascia, peritoneum) are divided through a vertical midline incision extending from the xiphoideum to the pubis with a few centimeters of fascia left intact at both ends to facilitate subsequent closure or late reconstruction. Alternatively, a transverse bilaterally extended incision few centimeters below the costal margins can be used to perform a fullthickness laparostomy [5] (Fig. 1). A third method utilizes

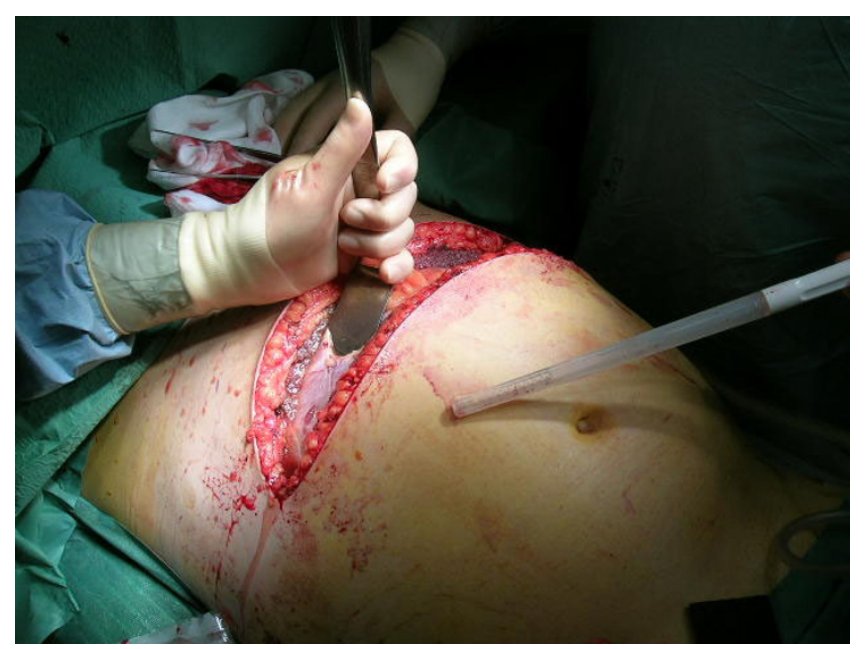

Figure I

Decompressive transverse laparostomy. three short horizontal skin incisions to perform a subcutaneous anterior abdominal fasciotomy at the linea alba (SLAF) with the peritoneum left intact [6].

\section{IAP decrease after full-thickness laparostomy}

All techniques have been shown to reduce IAP. The majority of evidence is based on experience from patients with SAP. In a collective of analysis of 161 patients undergoing surgical decompression via midline laparostomy, the mean reported IAP before surgical decompression was $34.6 \mathrm{mmHg}$ and fell to $15.5 \mathrm{mmHg}$ after decompression $(\mathrm{p}<0.001)$ [4]. The experience with transverse laparostomy is still scarce, but a case report showed the decrease from $23 \mathrm{mmHg}$ to $10 \mathrm{mmHg}$ [5].

\section{IAP decrease after fasciotomy}

The original report of two patients utilizing the SLAF method showed a decrease of IAP from $30 \mathrm{mmHg}$ to 14 mmHg and $35 \mathrm{mmHg}$ to $23 \mathrm{mmHg}$, respectively [6]. Another report showed a decrease from $27 \mathrm{mmHg}$ to 11 mmHg [7]. In analyzing the first 10 patients with SAP undergoing SLAF at the Meilahti hospital in Helsinki, the mean preoperative IAP was 31 (range 23-45) $\mathrm{mmHg}$ and fell to $20(10-33) \mathrm{mmHg}$ immediately postoperatively with a mean decrease of 10 (2-17) $\mathrm{mmHg}$ [Leppäniemi A, unpublished data]. The initial decompressive effect was deemed sufficient in 7 patients, out of whom 2 developed recurrent ACS and underwent completion laparostomy.

\section{Selection of decompressive method}

Because there are no randomized studies comparing different surgical decompression techniques, the selection of the technique has to be individualized and based on common sense and weighing the pros and cons of each technique. The midline laparostomy is relatively safe, easy to perform and nearly always effective. Although early complications, such as intestinal fistulas, have been greatly reduced with careful management and better understanding of the open abdomen, there is a high risk of persistent open abdomen requiring split-thickness skin grafting and delayed reconstruction of the abdominal wall (planned hernia approach). Recently, the use of temporary mesh to facilitate gradual fascial closure has decreased the planned hernia rate.

Transverse laparostomy seems to be effective, is little more time-consuming and could have a higher rate of fascial closure. In addition, same principles of managing the open abdomen can be applied without additional equipment. The major disadvantage is the loss of abdominal and back extensor muscle functions, if fascial closure can not be achieved. This would require complex reconstruction procedures including innervated free flaps that not only restore continuity but also the functional integrity of the abdomen [8]. 
SLAF is effective in about $50-70 \%$, prevents open abdomen and its related complications, but is always associated with a subsequent hernia. In the acute phase, the cost effectiveness due to lesser need of nursing care and reoperation resources is a significant advantage.

\section{Where to decompress}

From a surgical technical point of view, the best place to perform a decompressive operation is the operation theatre. It provides adequate aseptic conditions, lighting, equipment and personnel, and is ergonomically better for the surgeon. However, in urgent situations, decompression can and sometimes should be performed in the Intensive Care Unit (ICU). Even under less urgent conditions, a surgical team with proper equipment can go the ICU and perform the operation there without additional risk to the patient. The benefit of not requiring the transfer of a critically ill patient with multiple monitors and ongoing medications is obvious, and it also saves operation room time.

\section{Effects of surgical decompression on organ failure and outcome}

In a collective review of 250 patients undergoing midline laparostomy, decompression had an overall positive effect on hemodynamic, respiratory and renal functions [4]. Central venous pressure and pulmonary artery pressure decreased, most likely caused by the direct effect of the decrease in IAP on the thoracic cavity. Cardiac function improved in the majority of the patients. There was an improvement in $\mathrm{PaO}_{2} / \mathrm{FIO}_{2}$ ratio and decrease in peak airway pressure, but the respiratory function remained severely impaired in most patients. Significant improvement in the urinary output was observed in all but two studies.

At our institution, among the 26 patients with SAP undergoing surgical decompression for ACS during the past 6 years, mostly a full-thickness midline laparostomy, there was no significant difference between pre- and postoperative organ dysfunctions score [Leppäniemi, unpublished data]. The $\mathrm{PaO}_{2} / \mathrm{FIO}_{2}$ ratio increased in $50 \%$ and decreased in $50 \%$ of the patients. Daily urinary output increased by $>200 \mathrm{ml}$ in 7 patients $(27 \%)$, and 3 patients avoided renal replacement therapy. The overall mortality rate was $46 \%$ with preoperative renal failure $(\mathrm{p}=0.045)$, lower preoperative IAP $(\mathrm{p}=0.039)$ and late (median 7 days) decompression $(\mathrm{p}=0.005)$ being associated with increased risk of death. It is noteworthy that all 8 patients undergoing surgical decompression more than 3 days post-admission died.

It seems that in most cases ACS is an early phenomenon requiring prompt action. If nonoperative techniques fail to provide significant decrease in the IAP and improvement in organ functions, surgical decompression should be performed without additional delay. As institutional experience in the management of ACS increases, the delay from onset of the problem to interventions tends to decrease.

\section{Complications of surgical decompression}

Although postoperative bleeding and infection can occur after any surgical procedure, they are rare after decompressive laparostomy. Recurrent ACS can develop after too eager closure attempts. However, the major source of morbidity is associated with the management and complications of the open abdomen.

\section{Management of the open abdomen}

In view of the complicated and often fatal outcome of patients ending up with a persistent open abdomen after multiple reoperations, often with entero-atmospheric fistulae and persistent infection, the "hostile abdomen" scenario, extreme caution and care should be administered when managing patients with open abdomen [9].

The ideal cover of the abdominal contents after leaving the abdomen open should protect the viscera, avoid fistulas, be easy to apply and remove, allow easy nursing care, should not damage the fascia or the skin, be readily available and inexpensive, and maintain the abdominal domain. In addition, the preservation of the accessibility to the abdominal cavity and the feasibility of gradual closure of the abdominal wall are important.

The easiest method to cover the abdominal viscera after decompression is a plastic silo (Bogota bag) which is inexpensive, readily available and which preserves the intact fascia when sutured to the skin edges. However, because the plastic silo or some other form of temporary abdominal closure allows the fascial edges to retract laterally, the abdominal cavity looses part of its volume resulting in difficult fascial closure under significant tension especially, if the closure is delayed beyond the first week.

The vacuum pack introduced in 1995 utilizes a polyethylene sheet tucked between the parietal peritoneum and the bowel, thus preventing the formation of adhesions between the abdominal wall and the bowel [10]. In 2001 the commercial vacuum-assisted wound management technique was introduced into everyday practise [11]. It helps the nursing care, but it has not been shown to be superior to various "self-made" applications used worldwide [12] (Fig. 2). Nevertheless, even in the management of the most severe complication of the open abdomen, the exposed enteric fistula, vacuum-assisted wound management is able to control the fistula secretion allowing the wound around it to heal $[13,14]$.

Recently, a technique combining vacuum-dressing and a temporary mesh has been described [15]. It allows the 


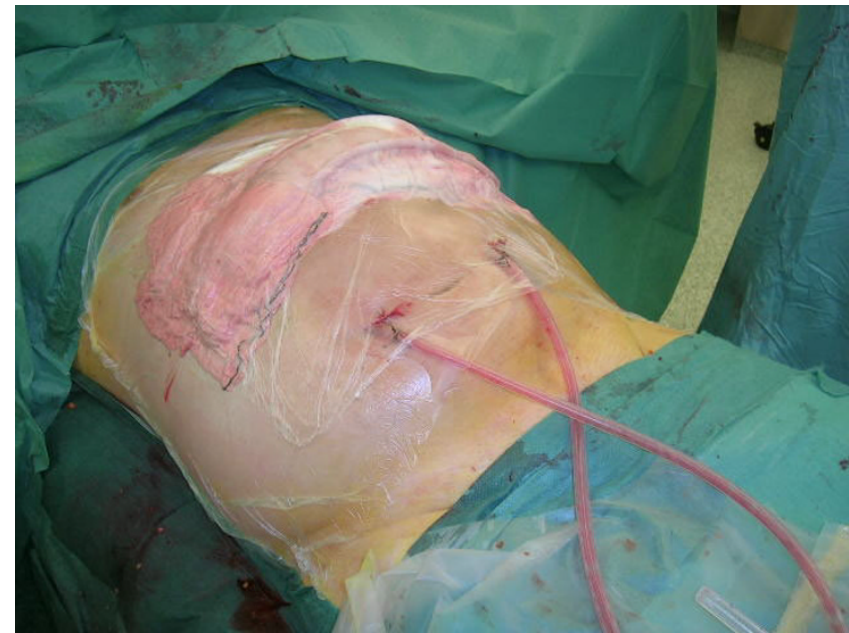

Figure 2

Covering transverse laparostomy with "self-made" negative pressure dressing.

gradual approximation of the fascial edges at every dressing change and eventual removal of the mesh and primary fascial closure (Fig. 3).

\section{Primary fascial closure}

Once the ACS has been treated, the most important aim is to achieve primary fascial closure as soon as possible without causing recurrent ACS or other complications associated with premature closure [16]. If the infection source has been controlled and even if a relaparotomy might be needed in the near future, every effort should be made to achieve primary fascial closure during the initial hospitalization period and avoid the significant morbidity associ-

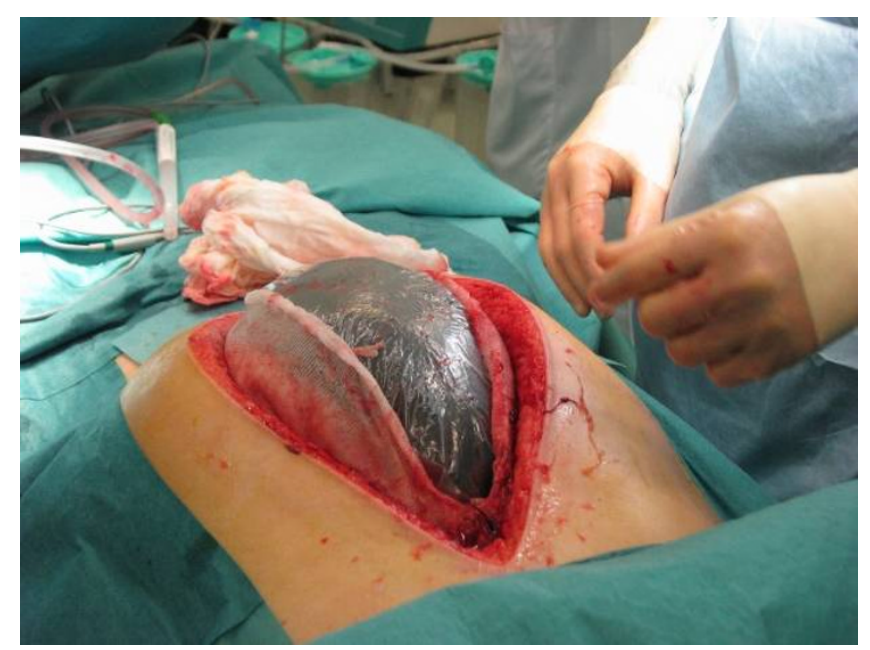

Figure 3

Vacuum-dressing with temporary mesh being changed. ated with leaving the abdomen open for delayed reconstruction. Gradual fascial closure, often meshassisted, seems to be the best currently available technique, but other possibilities, such as the components separation technique at an early stage [17], or fascial closure with a mesh prosthesis can be considered under favourable conditions (no infection, enough skin to cover the prosthesis). However, if primary fascial closure is not possible, an early decision to resort to the planned hernia strategy is a good option.

\section{Planned hernia}

A planned hernia approach aims at skin coverage with subsequent delayed abdominal wall reconstruction. The skin closure is most often achieved with autologous splitthickness skin grafting over the exposed bowel. Instead of allowing the bowel surface to granulate before skin grafting, early application of the skin graft over the bowel seems to enhance the tuning down of the inflammatory process sustained by the large raw surface, and to make the subsequent reconstruction process easier.

The conditions favouring a planned hernia strategy include the inability to reapproximate the retracted abdominal wall edges, sizeable tissue loss, risk of tertiary abdominal compartment syndrome, inadequate infection source control, anterior enteric fistula and poor nutritional status of the patient. One of the factors that must also be taken into account is the type of the abdominal wall defect, especially if it is the result of decompressive laparostomy. A midline laparostomy covered with skin graft is relatively easily tolerated, whereas one from transverse laparostomy might cause serious disability due to the loss of abdominal and back extensor muscle functions, if fascial closure can not be achieved [6]. This might require complex reconstruction procedures to restore not only continuity but also the functional integrity of the abdomen [5]. If abdominal decompression has been achieved with the SLAF technique, the subsequent hernia can be corrected in a standard fashion utilizing prosthetic mesh or the components separation technique.

The maturation of the skin graft requires about 9-12 months, after which the grafted skin can be easily removed from the bowel surface without additional iatrogenic lesions. Large abdominal wall defects can be reconstructed with pedicular or microvascular flaps. The most commonly used is the tensor fascia lata (TFL)-flap [18]. With smaller defects, the components separation technique or a mesh repair can be also used for late repair provided that there is enough original skin for skin closure.

\section{Conclusion}

IAH is a recently popularized clinical entity that is easily recognized, when suspected, using intravesicular measurement of IAP. The clinically significant manifestation, 
ACS, requires prompt attempts to reduce IAP starting with nonoperative measures. If these are insufficient, surgical decompression is warranted. The choice of the decompressive technique must be individualized. If a full-thickness laparostomy is performed, primary fascial closure should be attempted during the initial hospitalization period. If not possible, early resort to the planned hernia strategy and subsequent abdominal wall reconstruction are the best options provided that sufficient expertise is available.

\section{Competing interests}

The author declares that they have no competing interests.

\section{Consent section}

Written informed consents were obtained from the patients for publication of this review and accompanying images. Copies of the written consents are available for review by the Editor-in-Chief of this journal.

\section{References}

I. Malbrain MLNG, Cheatham ML, Kirkpatrick A, Sugrue M, Parr M, de Waele J, Balogh Z, Leppäniemi A, Olvera C, Ivatury R, D'Amours S, Wendon J, Hillman K, Johansson K, Kolkman K, Wilmer A: Results from International Conference of Experts on intra-abdominal hypertension and abdominal compartment syndrome. $I$. Definitions. Intensive Care Med 2006, 32:1722-1732.

2. Cheatham ML, Malbrain MLNG, Kirkpatrick A, Sugrue M, Parr M, De Waele J, Balogh Z, Leppäniemi A, Olvera C, Ivatury R, D'Amours S, Wendon J, Hillman K, Wilmer A: Results from International Conference of Experts on intra-abdominal hypertension and abdominal compartment syndrome. II. Recommendations. Intensive Care Med 2007, 33:95I-962.

3. De laet I, Malbrain MLNG: ICU management of the patient with intra-abdominal hypertension: what to do, when and to whom? Acta Clin Belg Suppl 2007:190-199.

4. De Waele IJ, Hoste EA, Malbrain ML: Decompressive laparotomy for abdominal compartment syndrome - a critical analysis. Crit Care 2006, 10:R5I.

5. Leppäniemi A, Mentula P, Hienonen P, Kemppainen E: Transverse laparostomy is feasible and effective in the treatment of abdominal compartment syndrome in severe acute pancreatitis. World J Emerg Surg 2008, 3:6.

6. Leppäniemi A, Hienonen P, Siren J, Kuitunen A, Lindström $O$, Kemppainen $E$ : Treatment of abdominal compartment syndrome with subcutaneous anterior abdominal fasciotomy in severe acute pancreatitis. World J Surg 2006, 30:1922-1924.

7. Cheatham M, Fowler J, Pappas P: Subcutaneous linea alba fasciotomy: a less morbid treatment for abdominal compartment syndrome. Am Surg 2008, 74:746-749.

8. Pushpakumar SB, Wilhelmi BJ, van-Aalst VC, Banis JC Jr, Barker JH Abdominal wall reconstruction in a trauma setting. Eur $J$ Trauma Emerg Surg 2007, 33:3-13.

9. Leppäniemi $A$ : The hostile abdomen - a systematic approach to a complex problem. Scand J Surg 2008, 97:218-219.

10. Brock WB, Barker DE, Burns RP: Temporary closure of open abdominal wounds: the vacuum pack. Am Surg 1995, 61:30-35.

11. Garner GB, Ware DN, Cocanour CS, Duke JH, McKinley BA, Kozar RA, Moore FA: Vacuum-assisted wound closure provides early fascial reapproximation in trauma patients with open abdomens. Am J Surg 200I, 182:630-638.

12. Leppäniemi A: Open abdomen after severe acute pancreatitis. EurJ Trauma Emerg Surg 2008, 34:17-23.

13. Erdmann D, Drye C, Heller L, Wong MS, Levin SL: Abdominal wall defects and enterocutaneous fistula treatment with the Vacuum Assisted Closure (V.A.C.) system. Plast Reconstr Surg 200I, 108:2066-2068.
14. Becker HP, Willms A, Schwab R: Small bowel fistulas and the open abdomen. Scand J Surg 2007, 96:263-27I.

15. Petersson U, Acosta S, Björck M: Vacuum-assisted wound closure and mesh-mediated fascial traction - a novel technique for late closure of the open abdomen. World J Surg 2007, 3I:2133-2137.

16. Scott BG, Feanny MA, Hirshberg A: Early definitive closure of the open abdomen: a quiet revolution. Scand J Surg 2005, 94:9-14.

17. Ramirez OM, Ruas E, Dellon AL: Components separation method for closure of abdominal-wall defects: and anatomic and clinical study. Plast Reconstr Surg 1990, 86:519-526.

18. Lyle WG, Gibbs M, Howdieshell TR: The tensor fascia lata free flap in staged abdominal wall reconstruction after traumatic evisceration. J Trauma 1999, 46:519-522.
Publish with Biomed Central and every scientist can read your work free of charge

"BioMed Central will be the most significant development for disseminating the results of biomedical research in our lifetime. "

Sir Paul Nurse, Cancer Research UK

Your research papers will be:

- available free of charge to the entire biomedical community

- peer reviewed and published immediately upon acceptance

- cited in PubMed and archived on PubMed Central

- yours - you keep the copyright
BioMedcentral 